\title{
Progress in radiotherapy and expression of PD-L1 in esophageal cancer
}

\author{
Xiangyong Liu ${ }^{1}$ and Ying Sun ${ }^{2 *}$ \\ ${ }^{1}$ Department of Radiotherapy, Qilu Hospital of Shandong University, Qingdao, China \\ ${ }^{2}$ Department of Medical Oncology, Qilu Hospital of Shandong University, Qingdao, China
}

\begin{abstract}
Esophageal cancer is a malignant tumor with high morbidity and mortality in China, and the overall prognosis is poor. Finding new treatment methods is a hot topic at home and abroad. This article summarizes the relationship between pd-11 expression and clinical pathology and prognosis of esophageal cancer, the effect of radiotherapy on the expression of pd-11, and finally discusses the effect of radiotherapy on pd-1/pd-11 treatment. It is suggested that the up-regulation of pd-11 in the tumor microenvironment not only shows immunosuppression, but also is related to endogenous anti-tumor immune activity. The prognosis of patients is affected by both immunosuppression and activation. Radiotherapy can up-regulate the expression of pd-11 in esophageal squamous cell carcinoma, and radiotherapy combined with anti-pd-1/pd-11 treatment has synergistic effect. Then the mechanism behind it is complicated, and there are many signal transduction pathways involved, which needs further study.
\end{abstract}

\section{Introduction}

According to the latest data released by the National Cancer Center, the number of new cases of esophageal cancer in China in 2014 was 258,000 , ranking 6th in malignant tumors, and the number of deaths was 193,000, ranking fourth in all malignant tumors [1]. More than $90 \%$ of esophageal cancers are squamous cell carcinomas. Despite the use of surgery, radiotherapy, chemotherapy and other comprehensive treatments, the prognosis of advanced esophageal cancer is still very poor, the 5 -year survival rate is only $15 \%$ to $25 \%$ [2]. Therefore, researching new treatments to improve the prognosis of esophageal cancer is an urgent need at home and abroad. The emergence of immunological checkpoint inhibitors has brought milestones to immunotherapy. In recent years, immunological checkpoint inhibitors have been used to treat diseases such as non-small cell lung cancer, malignant melanoma, Hodgkin's lymphoma, and have shown surprising effects. However, the effectiveness of immunological checkpoint inhibitors alone is only about $20 \%$, and immunization combined with other anti-tumor treatments is currently a hot topic. Pd-11 expression was used as a predictor of immunological checkpoint inhibitors, while high expression of pd-11 was thought to be associated with radiation resistance [3].The relationship between radiotherapy of esophageal cancer and pd-11 expression is reviewed.

\section{Overview of pd-1 and pd-11}

In 1992, Ishida, et al. [4] first discovered PD-1 (CD279), a member of the CD28 family member type I transmembrane glycoprotein, including the extracellular amino acid head, transmembrane domain, and cytoplasmic region amino acid tail, which contains immunity. The receptor tyrosine-based swith motif (ITSM) and the immunoreceptor tyrosine-based inhibitory motif (ITIM) [5]. Mainly expressed in peripheral CD4+ T cells, CD8 $+\mathrm{T}$ cells, regulatory $\mathrm{T}$ cells, $\mathrm{B}$ cells, dendritic cells (DCs), activated monocytes, natural killer cells (NK cells) and natural killer $\mathrm{T}$ cells.PD-1 is produced when $\mathrm{T}$ cells are stimulated by inflammation, and binds to their ligands in peripheral tissues mainly composed of tumors and infections [6]. The ligands of $\mathrm{PD}-1$ are $\mathrm{PD}-\mathrm{L} 1$ and $\mathrm{PD}-\mathrm{L} 2$, but only $\mathrm{PD}-\mathrm{L} 1$ is expressed in peripheral tissues, so PD-1 mainly acts on PD-L1 in the periphery.PD-L1 (CD274) belongs to the $\mathrm{B} 7$ superfamily and is also a type I transmembrane glycoprotein. It contains an $\mathrm{IgV}$-like region, an $\mathrm{IgC}$-like region, an amino acid intracellular region, and a transmembrane hydrophobic region. Tumor cells, antigen presenting cells (APCs), activated T cells, activated $\mathrm{B}$ cells, monocytes, macrophages, dendritic cells DCs, thymic cortical epithelial cells, and placental trophoblast cells [7].

\section{Relationship between expression of PD-L1 and clinicopathology and prognosis of esophageal cancer}

The relationship between PD-L1 expression and clinical pathology and prognosis of esophageal cancer remains controversial. A retrospective study of 378 patients with T2-T4a esophageal squamous cell carcinoma showed that PD-L1 expression in esophageal squamous carcinoma cells was associated with clinical pathological parameters such as age, differentiation, stage, metastasis, and DFS. Multivariate COX analysis did not. It can be shown that PD-L1 is an independent prognostic factor [8]. T Konno-Kumagai, et al. [9] found that high expression of PD-L1 in metastatic lymph nodes of esophageal squamous cell carcinoma was significantly associated with adverse clinical outcomes. However, a study by Chen $\mathrm{K}$, et al.

${ }^{\star}$ Correspondence to: Ying Sun, MD, Department of Medical Oncology, Qilu Hospital of Shandong University, Qingdao, No.758, Hefei Road, Shibei District, Qingdao, Shandong-266000, China, Tel: 86018561811172; E-mail: sduxy@ foxmail.com

Key words: esophageal cancer, $P D-L 1$, radiotherapy

Received: March 20, 2019; Accepted: March 27, 2019; Published: March 29, 2019 
[10] on esophageal squamous cell carcinoma showed that PD-L1 expression was associated with factors such as upper esophageal location, well-differentiated tumor type, no lymph node metastasis, and early disease, indicating that PD-L1 expression is An indicator of low invasive tumor types. Considering that PD-L1 may be affected by radiotherapy and chemotherapy and targeted therapy, Chen $\mathrm{X}$, et al. [11] were divided into single surgery group, postoperative radiotherapy group and postoperative chemotherapy group according to postoperative treatment. The expression of $\mathrm{PD}-\mathrm{L} 1$ in each group was observed. Relationship with prognosis. Interestingly, the prognosis of $\mathrm{PD}-\mathrm{L} 1$ positive patients in the postoperative radiotherapy group was significantly improved. However, there was no significant correlation between PD-L1 expression and prognosis in the single or postoperative chemotherapy group, suggesting that PD-L1 positive may be a good predictor of radiotherapy. Two studies in Japan found that PD-L1 expression improved the prognosis of patients undergoing surgery alone and did not improve the prognosis of patients undergoing adjuvant chemotherapy. It may be because chemotherapy not only has a direct cytotoxic effect on tumor cells, but also affects the tumor immune system $[12,13]$. In different studies, the difference between PD-L1 expression status and clinicopathological factors and survival rate of esophageal squamous cell carcinoma may be due to the upregulation of PD-L1 in the tumor microenvironment not only as immunosuppression but also with endogenous anti-tumor immunity. Related to activity. The prognosis of patients is affected by both immunosuppression and activation.

\section{Effect of radiotherapy on the expression of pd-11}

\section{Radiotherapy can cause up-regulation of pd-11 expression}

Radiotherapy is widely used in the treatment of primary and metastatic tumors. The biological response of a tumor to radiation includes DNA damage, regulation of signal transduction, and changes in the tumor microenvironment. Lim, et al. [14] compared the expression of PD-L1 after neoadjuvant chemoradiotherapy with esophageal squamous cell carcinoma and found that radiotherapy and chemotherapy can up-regulate PD-L1 expression. Ronan, et al. [15] also found that radiotherapy can up-regulate PD-L1 expression in a rat model of esophageal adenocarcinoma in a dose-dependent manner. Similar results were observed in other tumors. Radiotherapy increased PD-L1 expression in the head and neck squamous cell carcinoma cell line, and the effect on PD-L1 expression was dose-dependent [16]. High-dose radiotherapy can up-regulate PD-L1 expression [17] in pancreatic ductal adenocarcinoma. The expression of PD-L1 was significantly increased after neoadjuvant chemoradiotherapy for rectal cancer [18].

\section{Overview of pd-11 up-regulation related signaling pathways}

PD-L1 is considered to be an important mediator of epithelialmesenchymal transition (EMT) in certain human cancer tissues such as lung cancer, colorectal cancer, and head and neck cancer. The expression of PD-L1 in EMT-positive tumor samples was higher than that in EMT-negative tumor samples.PD-L1 promotes EMT and the role of tumor stem cell initiation and maintenance. PD-L1 overexpression enhances mesenchymal markers (N-cadherin, ZeB1 and Vimentin), resulting in EMT phenotype of ECA-109 cells [19]. A study by Alsulinman, et al. [20] suggested that the induction of epithelial mesenchymal transition in human breast cancer epithelial cells mainly up-regulates the expression of PD-L1 by activating the PI3K/Akt pathway. Another study found that PD-L1 expression induces epithelial mesenchymal transition [21] in renal cell carcinoma by activating the transcription factor SREBP-1C.A study by Aung K, et al. [22] found that inhibition of GSK-3 $\beta$ induced EMT phenotype and up-regulated PD-L1 expression. EMT-transformed tumor cells have the ability to induce $\mathrm{T}$ cell apoptosis.

Studies have shown that epidermal growth factor receptor (EGFR) activation up-regulates PD-L1 levels in lung cancer patients, a finding that links the EGFR pathway to PD-L1 expression [23]. Hoi, et al. [24] found that PD-L1 expression and EGFR and ERK activation were significantly induced in esophageal squamous carcinoma cell lines after standard chemotherapy, confirming that up-regulation of PDL1 by esophageal squamous cell carcinoma after chemotherapy is via EGFR/ERK signaling pathway Adjustment. A study by Zhang W, et al. [25] showed that PD-L1 expression was significantly increased in an EGFR-dependent manner by activating EGFR signaling, and that PD-L1 expression decreased dramatically when EGFR signaling was blocked. The study concluded that the upregulated expression of PDL1 is not associated with the EGFR-STAT3 signaling pathway, but may be affected by the EGFR-PI3K-AKT, EGFR-Ras-Raf-Erk, and EGRPLC- $\gamma$ signaling pathways.

Zhang P, et al. [26] found that PD-L1 and IL-6 were significantly elevated in cisplatin-resistant head and neck squamous carcinoma cells, and anti-IL-6/STAT3 antibodies partially inhibited IL-6 and PD-L1 expression. Shen, et al. [27] indicated that radiotherapy can up-regulate the expression of PD-L1 in tumor cells in a time- and dose-dependent manner. IL-6 signaling enhances the anti-radiation ability of lung cancer cells by promoting DNA repair after irradiation, suggesting that PD- The expression of L1 may be related to IL-6.

Jin, et al. [28] found that Rb hyperphosphorylation down-regulated the expression of PD-L1 induced by radiotherapy by inhibiting NF$\mathrm{kB}$ activity. Chen, et al. [29] showed that radiotherapy combined with toll-like receptor agonist CPG-ODN7909 can down-regulate PD-L1 expression by inhibiting NF-kB signaling pathway, suggesting that PDL1 expression may be regulated by NF-kB signaling pathway.

A recent study by Dovedi, et al. [3] has demonstrated that after radiotherapy, the expression of $\mathrm{PD}-\mathrm{L} 1$ in tumor cells is upregulated by interferon gamma produced by CD8+ $\mathrm{T}$ cells.In ALK-derived lung adenocarcinoma, PD-L1 expression [30] can be up-regulated by activating Stat 3 and hypoxia-inducible factor-1 $\alpha$ (HIF-1 $\alpha$ ) under normoxia and hypoxia. Low-dose fractionated radiotherapy resulted in up-regulation of tumor cell PD-L1 in a variety of isogenic mouse cancer models, and IFN production by CD8 $+\mathrm{T}$ cells mediates upregulation of PD-L1 in tumor cells [3]. Radiotherapy combined with gemcitabine up-regulated the expression of $\mathrm{PD}-\mathrm{L} 1$ in pancreatic cancer cells [17] by JAK/STAT1 dependent means. Kim, et al. [31] found that radiotherapy up-regulated PD-L1 expression via the PI3K/AKT and STAT3 pathways.

\section{Effect of radiotherapy on pd-1/pd-11 treatment}

Studies have shown that radiotherapy can trigger the recruitment and activation of antigen-presenting cells (APC) and initiate tumor antigen-specific $\mathrm{T}$ cell responses by releasing several damage-associated molecular patterns (DAMP) to regulate the host's immune response to tumor sites. In a mouse model, radiotherapy combined with anti-PD-L1 antibody synergistically enhances anti-tumor immunity by promoting CD8+ T cell infiltration and reducing accumulation of bone marrowderived suppressor cells (MDSCs) and tumor-infiltrating regulatory $\mathrm{T}$ cells (ITREGS) [32]. Lim, et al. [33] observed the expression of PD- 
L1 and the number of tumor-infiltrating lymphocytes in patients with rectal cancer before and after radiotherapy and chemotherapy. It was found that radiotherapy and chemotherapy can up-regulate PD-L1 expression and increase CD8+ T cell density. Radiotherapy up-regulates the expression of PD-L1 in tumor cells by IFN- $\gamma /$ STAT3 signaling, which is beneficial to the therapeutic effect of anti-PD-L1. Anti-PD-L1 combined with radiotherapy significantly inhibited tumor growth compared with anti-PD-L1 or radiotherapy alone. Radiosensitization after PD-L1 blockade is associated with decreased CD11b+GR1+ myeloid cell infiltration and enhanced CD45+CD8+ T cell infiltration, while T cell activation markers (including CD69, CD44 and Fasl) are upregulated, CD8:Treg ratio increase.Radiotherapy activates the immune system, triggering an anti-tumor immune response after cytotoxic death and release of an immune stimuli signal, thereby increasing the transport of $\mathrm{T}$ cells to the tumor [17]. Anti-PD-L1 combined with radiotherapy can significantly inhibit tumor growth, including increasing apoptosis, reducing tumor cell proliferation, and restoring $\mathrm{CD} 8+\mathrm{T}$ cell function. Anti-PD-L1 combined with radiation repairs $\mathrm{CD} 8+\mathrm{T}$ cell function to expand and restore its effector functions such as cytokine production and cell lysis [31]. Local radiotherapy induces the production of IFN- $\beta$, thereby increasing the expression of MHC I in drug-resistant tumor cells and restoring the reactivity of drugresistant tumors against PD1 treatment.Radiation is sensitive to PD-1 treatment by activating the IFN $\beta /$ IFNAR-MHC class I pathway [34]. In addition, in the melanoma, colorectal cancer and triple-negative breast cancer models, radiotherapy combined with PD-1 antibody treatment can improve its anti-tumor efficacy, providing a theoretical basis for the combination therapy strategy.

\section{Summary and outlook}

The overall prognosis of esophageal cancer is poor, and finding new treatment measures is a hot topic at home and abroad. The emergence of immunological checkpoint inhibitors has brought us a new dawn. However, immunological checkpoint inhibitors are inefficient and limit their use. In vitro tests initially showed synergistic effects between immunotherapy and radiotherapy. However, the specific mechanisms and the signal pathways involved are complex and require further research. Immunotherapy combined with radiotherapy is expected to bring more gospel to patients with esophageal cancer.

\section{References}

1. Chen W, Sun K, Zheng R (2018) Cancer incidence and mortality in China, 2014. Chinese journal of cancer research 30: 1-12.

2. Pennathur A, Gibson MK, Jobe BA, Luketich JD (2013) Oesophageal carcinoma. Lancet 381: 400-412. [Crossref]

3. Dovedi SJ, Adlard AL, Lipowska-Bhalla G (2014) Acquired resistance to fractionated radiotherapy can be overcome by concurrent PD-L1 blockade. Cancer research 74: 5458-5468.

4. Ishida Y, Agata Y, Shibahara K, Honjo T (1992) Induced expression of PD-1, a novel member of the immunoglobulin gene superfamily, upon programmed cell death. EMBO J 11: 3887-3895. [Crossref]

5. Sun H, Sun C, Xiao W (2014) Expression regulation of co-inhibitory molecules on human natural killer cells in response to cytokine stimulations. Cytokine 65: 33-41.

6. Boussiotis VA, Chatterjee P, Li L (2014) Biochemical signaling of PD-1 on T cells and its functional implications. Cancer J 20: 265-271.

7. Ceeraz S, Nowak EC, Noelle RJ (2013) B7 family checkpoint regulators in immune regulation and disease. Trends Immunol 34: 556-563. [Crossref]

8. Rong L, Liu Y, Hui Z (2019) PD-L1 expression and its clinicopathological correlation in advanced esophageal squamous cell carcinoma in a Chinese population. Diagn Pathol 14: 6.
9. Konnokumagai T, Fujishima F, Nakamura Y (2018) Programmed death-1 ligands and tumor infiltrating $\mathrm{T}$ lymphocytes in primary and lymph node metastasis of esophageal cancer patients. Dis Esophagus 1: 3.

10. Chen K, Cheng G, Zhang F (2016) Prognostic significance of programmed death-1 and programmed death-ligand 1 expression in patients with esophageal squamous cell carcinoma. Oncotarget 7: 30772-30780.

11. Jiang C, Zhu Y, Tang S (2019) High PD-L1 expression is associated with a favorable prognosis in patients with esophageal squamous cell carcinoma undergoing postoperative adjuvant radiotherapy. Oncol Lett 17: 1626-1634.

12. Tanaka K, Miyata H, Sugimura K (2016) Negative influence of programmed death1-ligands on the survival of esophageal cancer patients treated with chemotherapy. Cancer Sci 107: 726-733.

13. Wakita A, Motoyama S, Nanjo H (2017) PD-L1 Expression Is a Prognostic Factor in Patients with Thoracic Esophageal Cancer Treated Without Adjuvant Chemotherapy. Anticancer Res 37: 1433-1441.

14. Lim S H, Hong M, Ahn S (2016) Changes in tumour expression of programmed deathligand 1 after neoadjuvant concurrent chemoradiotherapy in patients with squamous oesophageal cancer. Eur J Cancer 52: 1-9.

15. Kelly RJ, Zaidi AH, Smith MA (2018) The Dynamic and Transient Immune Microenvironment in Locally Advanced Esophageal Adenocarcinoma Post Chemoradiation. Ann Surg 268: 992-999.

16. Schulz D, Stancev I, Sorrentino A (2019) Increased PD-L1 expression in radioresistant HNSCC cell lines after irradiation affects cell proliferation due to inactivation of GSK3beta. Oncotarget 10: 573-583.

17. Azad A, Yin Lim S, D'Costa Z (2017) PD-L1 blockade enhances response of pancreatic ductal adenocarcinoma to radiotherapy. EMBO Mol Med 9: 167-180.

18. Hecht M, Buttner-HeroldD M, Erlenbach-Wunsch K (2010) PD-L1 is upregulated by radiochemotherapy in rectal adenocarcinoma patients and associated with a favourable prognosis. Eur J Cancer 65: 52-60.

19. Chen L, Xiong Y, Li J (2017) PD-L1 Expression Promotes Epithelial to Mesenchymal Transition in Human Esophageal Cancer. Cell Physiol Biochem 42: 2267-2280.

20. Alsuliman A, Colak D, Al-Harazi O (2015) Bidirectional crosstalk between PD-L1 expression and epithelial to mesenchymal transition: significance in claudin-low breast cancer cells. Mol Cancer 14: 149.

21. Wang Y, Wang H, Zhao Q (2015) PD-L1 induces epithelial-to-mesenchymal transition via activating SREBP-1c in renal cell carcinoma. Med Oncol 32: 212.

22. Thar AM, Okayama H, Saito M (2018) Epithelial-mesenchymal transition-converted tumor cells can induce T-cell apoptosis through upregulation of programmed death ligand 1 expression in esophageal squamous cell carcinoma. Cancer Med 7: 3321-3330.

23. Chen N, Fang W, Zhan J (2015) Upregulation of PD-L1 by EGFR Activation Mediates the Immune Escape in EGFR-Driven NSCLC: Implication for Optional Immune Targeted Therapy for NSCLC Patients with EGFR Mutation. J Thorac Oncol 10: 910923

24. Ng H Y, Li J, Tao L (2018) Chemotherapeutic Treatments Increase PD-L1 Expression in Esophageal Squamous Cell Carcinoma through EGFR/ERK Activation. Transl Oncol 11: 1323-1333.

25. Zhang W, Pang Q, Yan C (2017) Induction of PD-L1 expression by epidermal growth factor receptor-mediated signaling in esophageal squamous cell carcinoma. Onco Targets Ther Volume 10: 763-771.

26. Zhang P, Liu J, Li W (2018) Lactoferricin B reverses cisplatin resistance in head and neck squamous cell carcinoma cells through targeting PD-L1. Cancer Med.

27. Shen M J, Xu L J, Yang L (2017) Radiation alters PD-L1/NKG2D ligand levels in lung cancer cells and leads to immune escape from NK cell cytotoxicity via IL-6-MEK/Erk signaling pathway. Oncotarget 8: 80506-80520.

28. Jin X, Ding D, Yan Y, Li H, Wang B, et al. (2019) Phosphorylated RB Promotes Cancer Immunity by Inhibiting NF-Î ${ }^{\circ} \mathrm{B}$ Activation and PD-L1 Expression. Mol Cell 73: 22-35. [Crossref]

29. Chen X, Zhang Q, Luo Y, Gao C, Zhuang X, et al. (2016) High-dose irradiation in combination with toll-like receptor 9 agonist $\mathrm{CpG}$ oligodeoxynucleotide 7909 downregulates PD-L1 expression via the NF- $\mathrm{I}^{\circ} \mathrm{B}$ signaling pathway in non-small cell lung cancer cells. Onco Targets Ther 9: 6511-6518. [Crossref]

30. Koh J, Jang J, Keam B (2016) EML4-ALK enhances programmed cell death-ligand 1 expression in pulmonary adenocarcinoma via hypoxia-inducible factor (HIF)-1alpha and STAT3. Oncoimmunology 5: e1108514. 
31. Kim K, Kim J, Lee S J (2017) Radiation improves antitumor effect of immune checkpoint inhibitor in murine hepatocellular carcinoma model. Oncotarget 8: 41242 41255 .

32. Gong X, Li X, Jiang T (2017) Combined Radiotherapy and Anti-PD-L1 Antibody Synergistically Enhances Antitumor Effect in Non-Small Cell Lung Cancer. $J$ Thorac Oncol 12: 1085-1097.
33. Lim YJ, Koh J, Kim S, Jeon SR, Chie EK, et al. (2017) Chemoradiation-Induced Alteration of Programmed Death-Ligand 1 and CD8+ Tumor-Infiltrating Lymphocytes Identified Patients With Poor Prognosis in Rectal Cancer: A Matched Comparison Analysis. Int J Radiat Oncol Biol Phys 99: 1216-1224. [Crossref]

34. Wang X, Schoenhals JE, Li A, Valdecanas DR, Ye H, et al. (2017) Suppression of Type I IFN Signaling in Tumors Mediates Resistance to Anti-PD-1 Treatment That Can Be Overcome by Radiotherapy. Cancer Res 77: 839-850. [Crossref]

Copyright: (2019 Liu X. This is an open-access article distributed under the terms of the Creative Commons Attribution License, which permits unrestricted use, distribution, and reproduction in any medium, provided the original author and source are credited. 